\title{
Article \\ A Machine Learning Method for the Quantitative Detection of Adulterated Meat Using a MOS-Based E-Nose
}

\author{
Changquan Huang ${ }^{1}$ (D) and $\mathrm{Yu} \mathrm{Gu}^{1,2,3,4, * \text { (D) }}$
}

1 College of Information Science and Technology, Beijing University of Chemical Technology, Beijing 100029, China; huangchangquan@mail.buct.edu.cn

2 Beijing Advanced Innovation Center for Soft Matter Science and Engineering, Beijing University of Chemical Technology, Beijing 100029, China

3 Guangdong Province Key Laboratory of Petrochemical Equipment Fault Diagnosis, Guangdong University of Petrochemical Technology, Maoming 525000, China

4 Department of Chemistry, Institute of Inorganic and Analytical Chemistry, Goethe-University, Max-von-Laue-Str. 9, 60438 Frankfurt, Germany

* Correspondence: guyu@mail.buct.edu.cn

check for updates

Citation: Huang, C.; Gu, Y. A Machine Learning Method for the Quantitative Detection of Adulterated Meat Using a MOS-Based E-Nose. Foods 2022, 11, 602. https://doi.org/10.3390/ foods11040602

Academic Editor: Silvia Grassi

Received: 11 January 2022

Accepted: 17 February 2022

Published: 20 February 2022

Publisher's Note: MDPI stays neutral with regard to jurisdictional claims in published maps and institutional affiliations.

Copyright: (C) 2022 by the authors. Licensee MDPI, Basel, Switzerland. This article is an open access article distributed under the terms and conditions of the Creative Commons Attribution (CC BY) license (https:// creativecommons.org/licenses/by/ $4.0 /)$.

\begin{abstract}
Meat adulteration is a global problem which undermines market fairness and harms people with allergies or certain religious beliefs. In this study, a novel framework in which a onedimensional convolutional neural network (1DCNN) serves as a backbone and a random forest regressor (RFR) serves as a regressor, named 1DCNN-RFR, is proposed for the quantitative detection of beef adulterated with pork using electronic nose (E-nose) data. The 1DCNN backbone extracted a sufficient number of features from a multichannel input matrix converted from the raw E-nose data. The RFR improved the regression performance due to its strong prediction ability. The effectiveness of the 1DCNN-RFR framework was verified by comparing it with four other models (support vector regression model (SVR), RFR, backpropagation neural network (BPNN), and 1DCNN). The proposed 1DCNN-RFR framework performed best in the quantitative detection of beef adulterated with pork. This study indicated that the proposed 1DCNN-RFR framework could be used as an effective tool for the quantitative detection of meat adulteration.
\end{abstract}

Keywords: meat adulteration; electronic nose; one-dimensional convolutional neural network; random forest regressor

\section{Introduction}

Meat is one of the best nutritional sources of protein for humans and is consumed worldwide due to its highly appreciated taste [1]. A recent report issued by the Organization for Economic Cooperation and Development and Food and Agriculture Organization (OECD-FAO) revealed that the average annual global meat consumption surpassed 327 million tons (carcass weight equivalent) from 2018 to 2020 [2]. Due to differences in prices, unethical producers sometimes blend expensive meat with lower priced meat, such as by supplementing beef with pork to increase profits [3]. An economic loss of USD 45.6 million occurred in Europe due to beef products being adulterated with horse meat [4]. Besides the economic loss, the illegal activity of fraudulent substitution also raises serious concerns about food safety, public health, religion, and ethics [5]. Therefore, it is important to develop a reliable method for the detection of adulterated meat.

To date, the technologies that have been used in the detection of meat adulteration include biology-based, chemistry-based, and spectroscopy-based methods. Biology-based technologies have included polymerase chain reaction (PCR) [6], polymerase chain reactionrestriction fragment length polymorphism (PCR-RFLP) [7], loop-mediated isothermal amplification (LAMP) [8], and enzyme-linked immunosorbent assay (ELISA) [9]. Although 
these methods have proven to be reliable, specific, and sensitive, biology-based technologies are time-consuming, expensive, and require complex laboratory procedures to be performed by skilled personnel $[10,11]$. Chemistry-based technologies, such as gas chromatography mass spectrometer (GCMS) [12] and liquid chromatography-tandem mass spectrometer (LC-MS/MS) [13], have been proposed for halal verification and the accurate identification of meat products. Chemistry-based technologies are reliable and precise in the identification of adulterated meat. However, they require complex extractions and have long analysis times which significantly limit their widespread use [14]. For spectroscopybased technologies, near-infrared spectroscopy (NIRS) [15], Raman spectroscopy (RS) [16], and hyperspectral imaging (HSI) [17] have been shown to be useful for detecting meat adulteration. However, the complex spectroscopy data requires a high degree of technical expertise to analyze. Due to the various drawbacks of these methods, it is imperative to develop a fast, precise, simple, and low-cost method for the detection of meat adulteration.

An electronic nose (E-nose) is a chemical measurement system used to measure the chemical properties of volatile gases and has been widely applied to detect the quality and safety of food due to its fast speed, high reliability, simple operation, and relatively low cost $[18,19]$. In recent years, reports of detecting meat adulteration using an E-nose have been increasing. Tian et al. [14] built a backpropagation neural network (BPNN) model for the prediction of pork content in minced mutton using a metal oxide semiconductor (MOS)-based E-nose and obtained a root mean square error (RMSE) of $5.26 \%$ on a test set. Han et al. [20] proposed a BPNN model for detecting pork adulteration in beef using a low-cost E-nose based on colorimetric sensors and got an RMSE of 0.147. Sarno et al. [21] proposed an optimized E-nose system using an optimized support vector machine (SVM) for the identification of pork in beef products and got an accuracy of $98.10 \%$. To the best of our knowledge, most studies that have utilized E-nose devices have achieved good results in qualitative detection of meat adulteration; however, few studies have demonstrated reliable quantitative detection. An important reason underlying the imperfect performance in quantitative detection is that these studies have depended on manually selecting and extracting features. These manual operations not only burden the user with complex tasks but are also likely to lose valuable information.

The present work therefore aimed to propose a precise method for the quantitative detection of minced beef adulterated with pork. A high-efficiency framework (1DCNN-RFR) consisting of a 1DCNN backbone and an RFR is proposed for the quantitative detection of meat adulteration. The 1DCNN backbone is a powerful feature extractor that automatically mines the volatile compound information of meat samples. The RFR is employed as the regressor to strengthen the anti-overfitting ability, instead of the fully connected layer, for predicting adulterated proportions.

\section{Materials and Methods}

\subsection{Meat Sample}

In this study, fresh beef and pork satisfying Chinese national food safety standards [22] were purchased from a Carrefour supermarket in Beijing, China, and transported to the author's laboratory in $20 \mathrm{~min}$. Once in the laboratory, the fat and connective tissue were removed and the beef and pork were minced for 1 minute using a commercial blender (ZG-L805, Guangdong Zhigao Co. Ltd., Foshan, China). The minced beef was adulterated by mixing it with minced pork at seven distinct proportions by weight $(0 \%, 10 \%, 20 \%$, $30 \%, 40 \%, 50 \%$, and $60 \%$ ). Adulterated proportions higher than $60 \%$ were not considered because they are easily identifiable by human senses.

To undertake a more comprehensive evaluation of the models, two independent measurements (Measurement $\mathrm{A}$ and Measurement $\mathrm{B}$ ) of sample gases were taken by the E-nose every day. For Measurement $\mathrm{A}$, meat samples were purchased and measured in the morning. For Measurement B, meat samples were purchased and measured in the afternoon. Every day, three samples of each adulterated proportion were prepared, making 21 samples ( 3 samples of each proportion $\times 7$ proportions) for both Measurement A and 
Measurement B. The daily operations for making experiment samples were same and lasted for 10 days. A total of 420 samples (10 days $\times 2$ measurements $\times 21$ samples) were measured. The details of the meat samples are shown in Tables 1 and 2.

Table 1. Details of the meat samples in Measurement A.

\begin{tabular}{cccc}
\hline No. & Adulterated Proportion & Measuring Time & Number of Samples \\
\hline Sa.P1 & $0 \%$ & Morning & $30(10$ days $\times 3$ samples $)$ \\
Sa.P2 & $10 \%$ & Morning & $30(10$ days $\times 3$ samples $)$ \\
Sa.P3 & $20 \%$ & Morning & $30(10$ days $\times 3$ samples $)$ \\
Sa.P4 & $30 \%$ & Morning & $30(10$ days $\times 3$ samples $)$ \\
Sa.P5 & $40 \%$ & Morning & $30(10$ days $\times 3$ samples $)$ \\
Sa.P6 & $50 \%$ & Morning & $30(10$ days $\times 3$ samples $)$ \\
Sa.P7 & $60 \%$ & Morning & $30(10$ days $\times 3$ samples $)$ \\
\hline
\end{tabular}

Table 2. Details of the meat samples in Measurement B.

\begin{tabular}{cccc}
\hline No. & Adulterated Proportion & Measuring Time & Number of Samples \\
\hline Sa.P1 & $0 \%$ & Afternoon & $30(10$ days $\times 3$ samples $)$ \\
Sa.P2 & $10 \%$ & Afternoon & $30(10$ days $\times 3$ samples $)$ \\
Sa.P3 & $20 \%$ & Afternoon & $30(10$ days $\times 3$ samples $)$ \\
Sa.P4 & $30 \%$ & Afternoon & $30(10$ days $\times 3$ samples $)$ \\
Sa.P5 & $40 \%$ & Afternoon & $30(10$ days $\times 3$ samples $)$ \\
Sa.P6 & $50 \%$ & Afternoon & $30(10$ days $\times 3$ samples $)$ \\
Sa.P7 & $60 \%$ & Afternoon & $30(10$ days $\times 3$ samples $)$ \\
\hline
\end{tabular}

\subsection{Data Collection by E-Nose}

In this study, a PEN3 E-nose (Airsense Analytics GmbH, Schwerin, Germany) was used to collect the E-nose data of the adulterated meat. The PNE3 E-nose has a sensor array with 10 different MOS sensors. The details of the sensors are listed in Table 3.

Table 3. Sensor array details [23].

\begin{tabular}{|c|c|c|}
\hline No. & Sensor & Main Performance \\
\hline 1 & W1C & Sensitive to aromatic compounds \\
\hline 2 & W5S & $\begin{array}{l}\text { High sensitivity to nitrogen oxides, broad } \\
\text { range sensitivity }\end{array}$ \\
\hline 3 & W3C & $\begin{array}{l}\text { Sensitive to ammonia and aromatic } \\
\text { compounds }\end{array}$ \\
\hline 4 & W6S & Sensitive mainly to hydrogen \\
\hline 5 & W5C & $\begin{array}{l}\text { Sensitive to alkanes and aromatic } \\
\text { components and less sensitive to polar } \\
\text { compounds }\end{array}$ \\
\hline 6 & W1S & $\begin{array}{c}\text { Sensitive to methane, broad range } \\
\text { sensitivity }\end{array}$ \\
\hline 7 & W1W & $\begin{array}{l}\text { Sensitive primarily to sulfur compounds } \\
\text { and many terpenes and organic sulfur } \\
\text { compounds }\end{array}$ \\
\hline 8 & W2S & $\begin{array}{l}\text { Sensitive to ethanol and less sensitive to } \\
\text { aromatic compounds }\end{array}$ \\
\hline 9 & W2W & $\begin{array}{l}\text { Sensitive to aromatic compounds and } \\
\text { organic sulfur compounds }\end{array}$ \\
\hline 10 & W3S & Highly sensitive to alkanes \\
\hline
\end{tabular}

All experiments were conducted in a single clean laboratory room (about 45 square meters) with the temperature and relative humidity were controlled at $25 \pm 1{ }^{\circ} \mathrm{C}, 50 \pm 2 \%$, respectively. Measurements of the sample gases were conducted in a well-ventilated 
location to reduce baseline fluctuations and interference from other gas molecules. The air in the working environment was filtered by two active charcoal filters (Filter 1 and Filter 2 in Figure 1) to produce the zero gas, which was used as the baseline in this study.

The workflow of E-nose data collection included a collection stage and cleaning stage, as shown in Figure 1. Before collecting E-nose data, the meat samples were placed in sealed $50 \mathrm{~mL}$ samplers for $3 \mathrm{~min}$ so that the volatile gases from the samples would fill the sampler airspace. During the collection stage, zero-point trim was first conducted via a $15 \mathrm{~s}$ automatic adjustment and calibration of the zero gas. The values relative to the zero-point values were recorded as a baseline. After the establishment of the baseline, the volatile gases from the meat samples were pumped into the sensor chamber following Arrow 1 in Figure 1 at a constant flow rate of $10 \mathrm{~mL} / \mathrm{s}$ where they contacted the MOS sensors. In this manner, the gas molecules were absorbed on the sensors' surfaces where they changed the sensors' conductivity through redox reactions with the sensors' active elements [23]. The sensors' conductivities stabilized when they were saturated. The sample gases were measured every $1 \mathrm{~s}$ for $100 \mathrm{~s}$ during the collection stage. During the cleaning stage, clean air from Filter 2 was pumped into the sensor chamber in the direction of Arrow 2 in Figure 1 to entirely remove the substances that had absorbed on the surface of the sensors. The cleaning stage lasted $30 \mathrm{~s}$. As a result, the measurement of each sample lasted 325 s. Figure 2 shows examples of response curves of the 10 sensors' during the collection stage for the 7 different proportions of adulterated meat. Each response curve represents the ratio of $G$ (the conductivity of the sensor when contacted by the sample's volatile gases) to G0 (the conductivity of the sensor when contacted by the zero gas). As shown in Figure 2, the response curves rose slowly and steadily from $0 \mathrm{~s}$ to $90 \mathrm{~s}$ and stabilized after $90 \mathrm{~s}$.

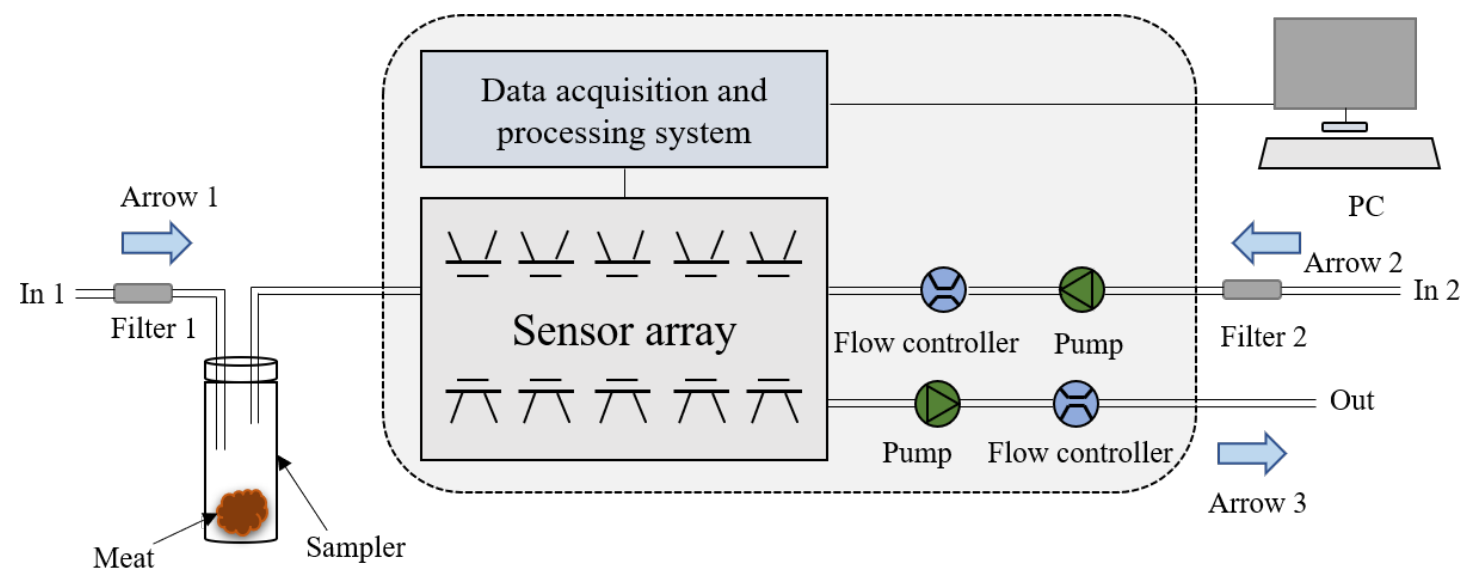

Figure 1. Schematic diagram of data collection using an E-nose.

\subsection{Dataset Settings}

The stable value (SV) is a vital and simple feature of the E-nose response signal which reflects the properties of the substances in the volatile gas and can be used by pattern recognition algorithms [24]. In this study, SVs (the values from $91 \mathrm{~s}$ to $100 \mathrm{~s}$ of the response signal) were used as the input data to the SVR, RFR, and BPNN. The response values from whole data collection stage (the values from $1 \mathrm{~s}$ to $100 \mathrm{~s}$ of the response signal) were used as the input data of the 1DCNN framework and the 1DCNN-RFR framework. The details of the datasets used in this study were as follows.

Dataset A: This dataset comprised a total of 210 samples (10 days $\times 3$ samples for each proportion $\times 7$ proportions) of Measurement A. For the SVR, RFR, and BPNN, the dataset could be expressed as a $2100 \times 10$ (number of sensors) matrix. For the $1 D C N N$ and $1 D C N N-R F R$ frameworks, the dataset could be expressed as a $210 \times 10 \times 100$ matrix.

Dataset B: This dataset comprised a total of 210 samples (10 days $\times 3$ samples for each proportion $\times 7$ proportions) of Measurement B. For the SVR, RFR, and BPNN, the 
dataset could be expressed as a $2100 \times 10$ (number of sensors) matrix. For the $1 D C N N$ and $1 D C N N-R F R$ frameworks, the dataset can be expressed as a $210 \times 10 \times 100$ matrix.

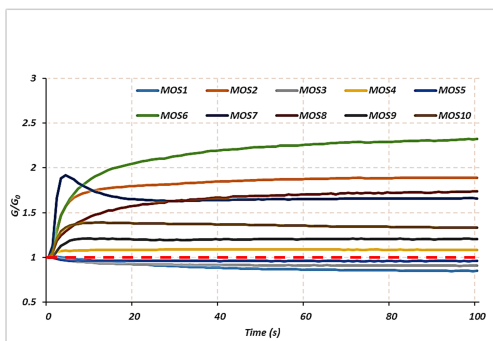

(a)

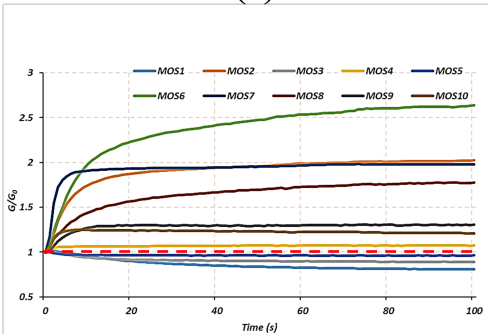

(d)

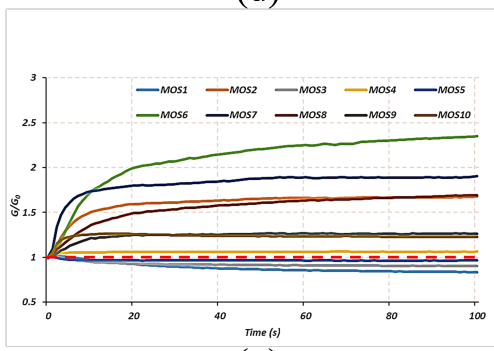

(g)

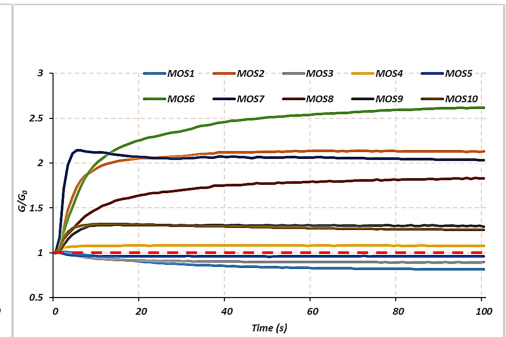

(b)

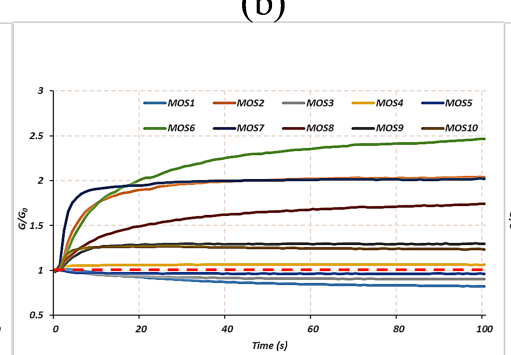

(e)

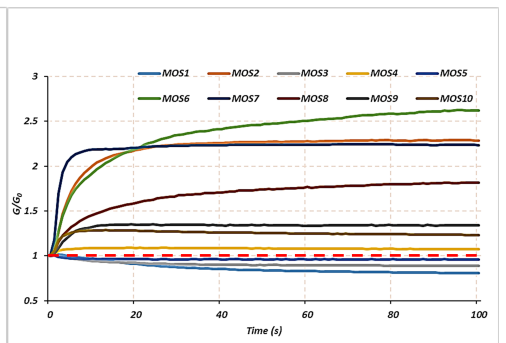

(c)

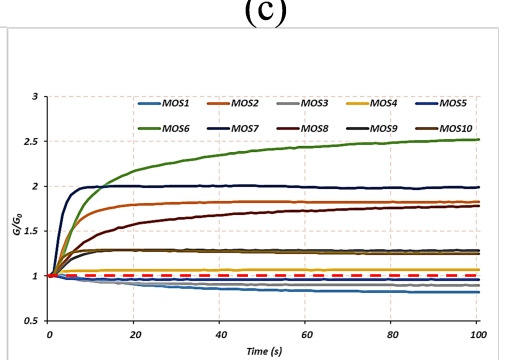

(f)

Figure 2. Example response curves of the 10 E-nose sensors (the baseline is marked with the red dotted line): (a) proportion of $0 \%$; (b) proportion of $10 \%$; (c) proportion of $20 \%$; (d) proportion of $30 \%$; (e) proportion of $40 \%$; (f) proportion of $50 \%$; (g) proportion of $60 \%$.

\subsection{Related Works}

\subsubsection{Principal Component Analysis (PCA)}

Principal component analysis (PCA) is a commonly utilized method for feature extraction [25]. PCA is mathematically defined as an orthogonal linear transformation that transforms data into a new coordinate system such that the greatest variance by some projection of the data comes to lie along the first coordinate (the first principal component), the second greatest variance along the second coordinate, and so on [26]. Generally, the first few principal components must contribute at least $85 \%$ of the variance, or else the PCA method would be considered unsuitable because too much of the original information would be lost [27]. The first few principal components to make up a cumulative contribution exceeding 95\% contain nearly all the information of the original data [24]. PCA is arguably the most popular multivariate statistical technique and has been applied in nearly all scientific disciplines [28].

\subsubsection{Convolutional Neural Network (CNN)}

The CNN is a supervised feed-forward deep learning network designed to process data that come in the form of multiple arrays [29]. Basically, CNN are composed of three types of layers: the convolutional, pooling, and fully-connected layers [30,31]. The convolutional layer is composed of several convolutional kernels which are used to compute different feature maps and the pooling layer merges semantically similar features into one and prevents overfitting. After several convolutional and pooling layers, there will be one 
or more fully connected layers which take all neurons from the previous layer and connect them to every single neuron of the current layer to generate global semantic information. CNNs have provided excellent performance solutions to various problems in image classification, object detection, games and decisions, and natural language processing [32].

\subsubsection{Random Forest (RF)}

Random forest (RF) is a combination of tree predictors such that each tree depends on the values from a random vector sampled independently and where all the trees in the forest have the same distribution [33]. The aim of the RF is to create a large number of uncorrelated decision tree models to produce more accurate predictions [34]. According to the strong law of large numbers, an increasing number of decision tree models leads to better generalizations and prevention of overfitting [35]. For the construction of the RF, N bootstrap samples are first drawn from the original training set (with replacement). Then, for each bootstrap sample, an unpruned classification or regression tree is grown with the following modifications: at each node, random sample $\mathrm{m}(\mathrm{m}<\mathrm{M})$ of the predictors (each sample contains $\mathrm{M}$ predictors) is taken and the best split from among those variables is chosen. The second step is repeated until the node can no longer be split without pruning. Finally, the generated decision trees are formed into a random forest that is used to classify or regress the new data [36,37]. Compared to other machine learning methods, RF has various advantages, including low complexity, fast computing speed, lower overfitting, etc. [38].

\subsubsection{Evaluation Metrics}

In this study, three evaluation metrics, including the coefficient of determination $\left(R^{2}\right)$, the root mean square error (RMSE), and the mean absolute error (MAE), were used to evaluate the regression performance of the four models and the proposed framework. The $R^{2}$ is usually presented as an estimate of the percentage of variance within the response variable explained by its (linear) relationship with the explanatory variables [39]. The RMSE represents the standard deviation of the differences between the predicted values and the observed values of the samples [19]. The MAE is defined as the average absolute difference between the predicted values and the observed values of the samples. The evaluation metrics are defined in Equations (1)-(3).

$$
\begin{gathered}
R^{2}=1-\frac{\sum_{i=1}^{n}\left(y_{i}-\widehat{y}_{i}\right)^{2}}{\sum_{i=1}^{n}\left(y_{i}-\bar{y}\right)^{2}} \\
R M S E=\sqrt{\frac{\sum_{i=1}^{n}\left(y_{i}-\widehat{y}_{i}\right)^{2}}{n}} \\
M A E=\frac{1}{n} \sum_{i=1}^{n}\left|y_{i}-\widehat{y}_{i}\right|
\end{gathered}
$$

where $n$ is the number of the samples in the training set or the test set; $y_{i}$ is the actual value of the ith sample; $\widehat{y}_{i}$ is the predicted value of the ith sample; and $\bar{y}$ is the mean of the actual value.

\section{Proposed Method}

Although the RFR has a low complexity and a lower likelihood of overfitting, its poor ability to extract a sufficient number of features may limit its performance. The 1DCNN is a type of CNN model [40] which only performs one-dimensional convolutions, giving them simpler structures and fewer parameters. Therefore, 1DCNN can save on computing resources and time [41]. Generally, a 1DCNN with the appropriate structure can mine sufficient features from the input data and with a flexible form. However, the 1DCNN requires more samples for training than traditional statistical models and the number of samples required may make it impractical for E-nose applications, where it may result in overfitting. Thus, a 1DCNN-RFR framework (presented in Figure 3a) consisting of a 
1DCNN backbone (presented in Figure 3b) and an RFR was developed to combine the advantages of the 1DCNN and RFR to predict the proportions of pork in adulterated minced beef.

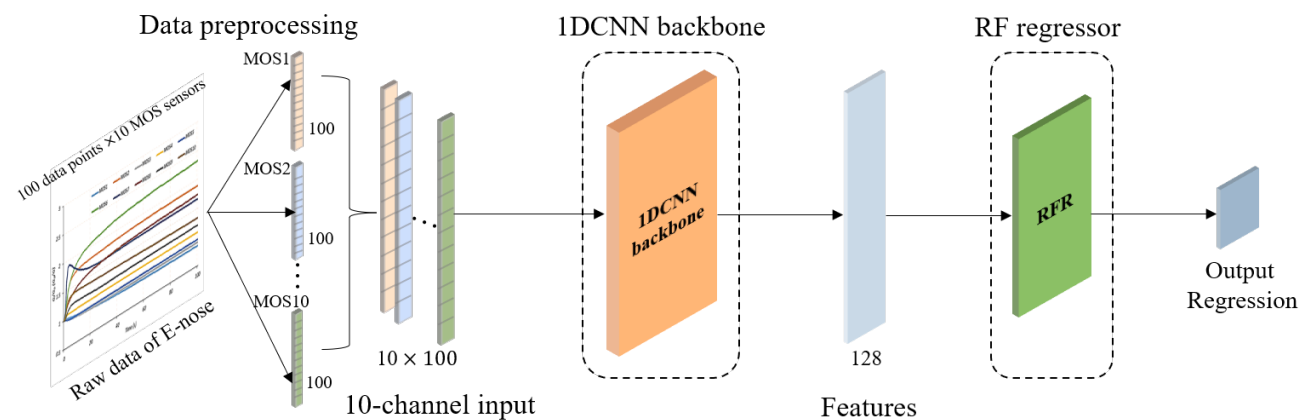

(a)

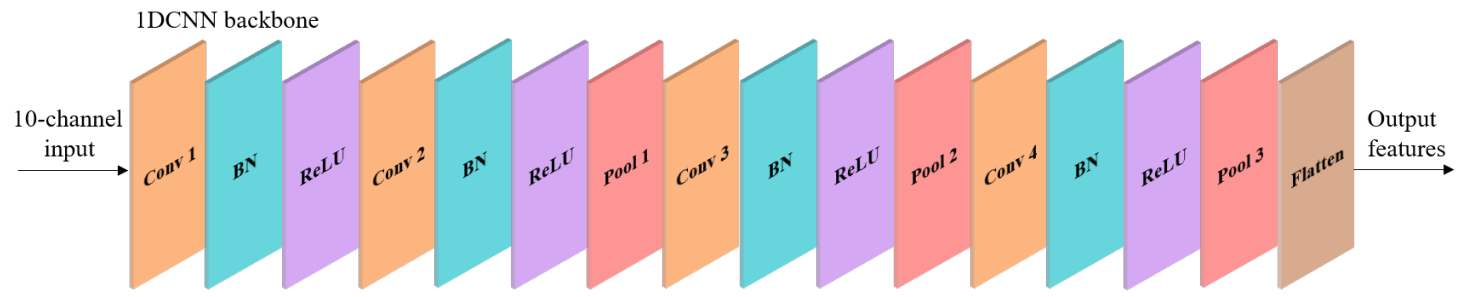

(b)

Figure 3. Schematic diagram of the proposed framework. (a) Structure of the proposed 1DCNN-RFR framework; (b) structure of the proposed 1DCNN backbone.

\subsection{Data Preprocessing}

In recent years, data preprocessing has increased in importance because data-mining algorithms require meaningful and manageable data so that they can correctly operate and provide useful knowledge, predictions, and descriptions. As Table 3 shows, the 10 MOS sensors exhibited strong cross-sensitivity because the sensitive substances of the sensors had some overlap. This is why E-nose data needs to be preprocessed before determining correlations between the sensors for the input of the 1DCNN backbone. Thus, the data preprocessing step is crucial to improving the prediction precision. To fit the multichannel input used in the 1DCNN model, the raw E-nose data were converted into a 10-channel input for the 1DCNN backbone. As shown in Figure 3a, the raw E-nose data formed a matrix with a size of $100 \times 10$, where 100 represents the 100 data points in the data collection stage (100 s) from each sensor and 10 represents the 10 MOS sensors. The 100 raw data points from each sensor were converted into a vectors with lengths of 100, and the 10 vectors of the sensors were concatenated into a $10 \times 100$ matrix as a 10-channel input of the 1DCNN backbone.

\subsection{Proposed $1 D C N N-R F R$ Framework}

In this study, a 1DCNN backbone consisting of four convolutional layers and three pooling layers was constructed to automatically extract features from the E-nose data. For all convolutional operations, the convolutional kernel size was 3. A batch normalization layer was used to accelerate convergence and improve the generalization ability of the operation. ReLU was selected as the activation function to increase the ability of nonlinear expression. In all downsampling operations, the stride was 2 and the filter size was 2 for the first two pooling layers (Pool 1 and Pool 2). For the last pooling layer (Pool 3), the stride was 1 and the filter size was 3 . A flattening layer came last, wherein a flattening transformation is applied to the tensor to convert the two-dimensional matrix of features into a vector that can be fed into the RFR. The specific design of the layers is shown in Table 4. The RFR, 
which consists of 100 random decision trees, is used to predict the adulterated proportions. Each tree is grown based on a resampling, using a regression tree procedure with a random subset of variables selected at each node.

Table 4. Details of the 1DCNN backbone.

\begin{tabular}{cccc}
\hline No. & Layer & Parameters & Outpur Size \\
\hline Input & Input & $/$ & $10 \times 100$ \\
Conv 1 & ConvBNReLU & 16@3, padding $=1$, stride $=2$ & $16 \times 50$ \\
Conv 2 & ConvBNReLU & 32@3, padding $=0$, stride $=1$ & $32 \times 48$ \\
Pool 1 & Max Pooling & Filter size $=2$, stride $=2$ & $32 \times 24$ \\
Conv 3 & ConvBNReLU & 64@3, padding $=1$, stride $=2$ & $64 \times 12$ \\
Pool 2 & Max Pooling & Filter size $=2$, stride $=2$ & $64 \times 6$ \\
Conv 4 & ConvBNReLU & 128@3, padding $=1$, stride $=2$ & $128 \times 3$ \\
Pool 3 & Avg Pooling & Filter size $=3$, stride $=1$ & $128 \times 1$ \\
Flatten & Flatten & $/$ & 128 \\
\hline
\end{tabular}

As shown in Figure 4, the 1DCNN-RFR framework workflow begins with the 1DCNN backbone automatically extracting features from the E-nose data, which are then fed to the RFR to output the adulterated proportions. An MSE loss function serves to calculate error and is minimized by the stochastic gradient descent (SGD) optimizer. A learning decay strategy with a fixed step size is used during training of the 1DCNN backbone. The batch size and the initial learning rate were set to 49 and 0.001 , respectively. After the training process of the framework is completed (solid line), the test samples are used to verify the performance of the trained framework (dotted line).

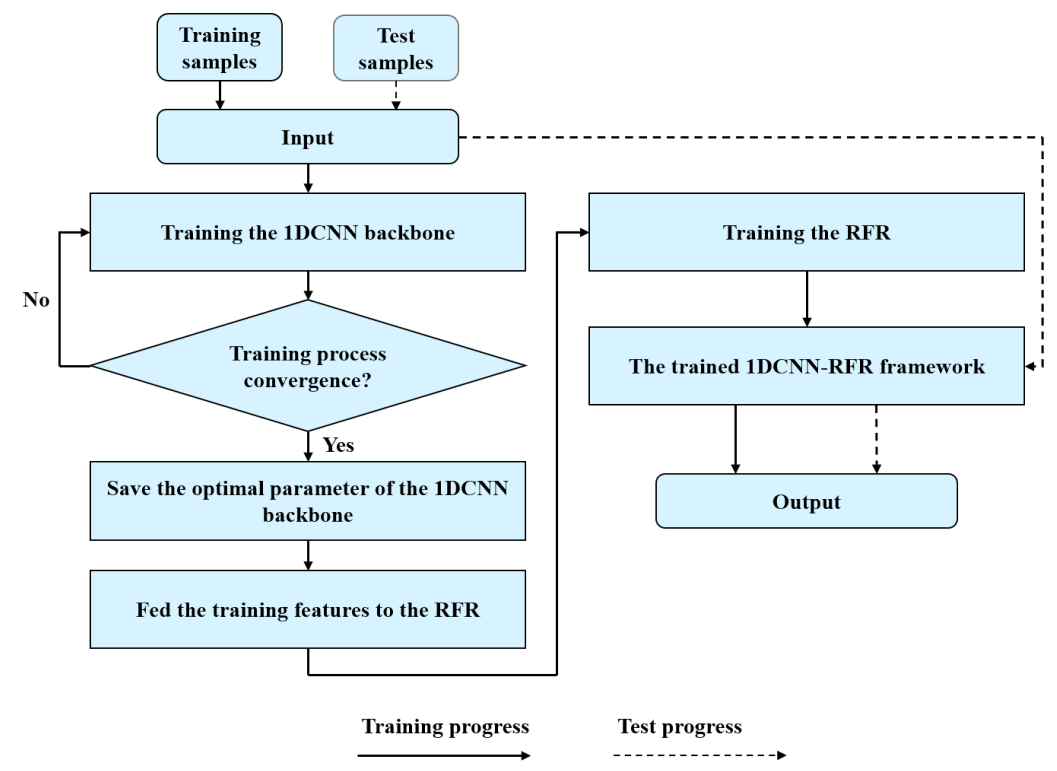

Figure 4. The workflow of the 1DCNN-RFR framework.

\section{Results and Discussion}

\subsection{Principal Component Analysis}

In pattern recognition, principal component analysis (PCA) is commonly used for feature extraction. In this study, the PCA was used to intuitively show the data distribution in a low-dimensional space by extracting meaningful information from the raw E-nose data. The SVs of 63 randomly selected meat samples (9 samples of each proportion $\times$ 7 proportions) were used in the PCA. The first three principal components contributed $92.76 \%$ of the variance and were selected to visualize the PCA results. The projections of the first three principal components are shown in Figure 5. The three axes represent the first principal component (PC 1), the second principal component (PC 2), and the third principal 
component (PC 3), which contributed 58.53\%, 26.40\%, and 7.83\%, respectively. As shown in Figure 5, the PCA was able to distinguish pure beef from adulterated meat. However, there was a large overlap between the adulterated meats of different proportions. Therefore, the PCA was unable to distinguish among meat samples with different proportions of adulteration.

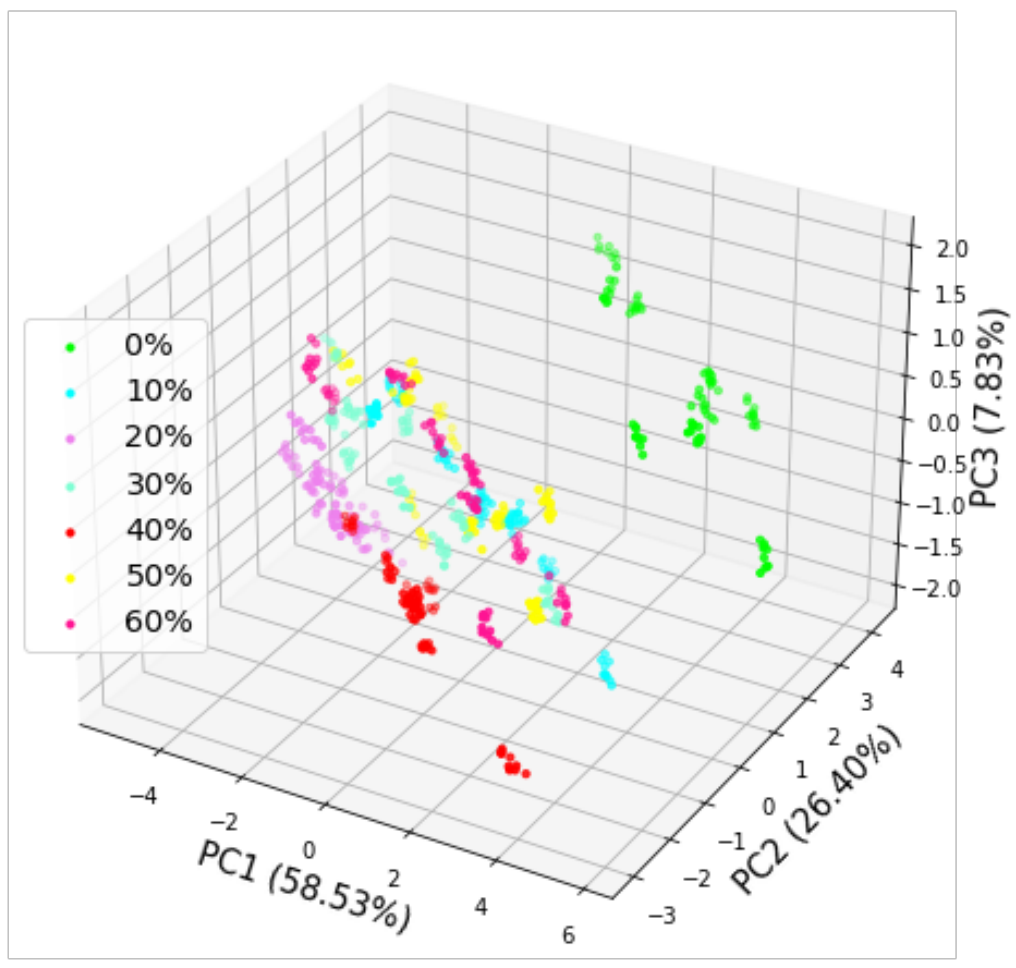

Figure 5. Projection of the first three principal components of the PCA of the meat samples.

\subsection{Comparing Five Models}

Support vector regression (SVR), as an extension of the support vector machines in regressions, is commonly applied in a variety of fields [42]. BPNN, as a known machine learning method, is proven to have great potential in rapid detection of meat adulteration [14,20]. Therefore, an SVR model with RBF kernel and a BPNN model were also examined in this study. The performances of the SVR model, RFR model, BPNN model, and 1DCNN framework were compared with that of the proposed 1DCNN-RFR framework using multiple evaluation metrics, i.e., $R^{2}$, RMSE, and MAE. The 1DCNN framework consisted of the $1 D C N N$ backbone and a 1DCNN regressor. The $1 D C N N$ regressor consisted of two fullyconnected layers with 64 and 32 neurons, respectively. ReLU and Sigmoid were selected as the activation functions for the first and second fully connected layers to strengthen the nonlinear expression ability of the 1DCNN regressor. A grid search method was used for identifying the best parameters of the five models via a three-fold cross-validation using the training set. The five models were programmed using the scikit learn library and the open-source PyTorch framework. Two experiments (Experiment A and Experiment B) with two datasets (Dataset A and Dataset B) were conducted to thoroughly compare the performances of the five models.

\subsubsection{Experiment A}

Dataset A was used in Experiment A, and was divided into two parts, namely the training set (data from the first 7 days, 147 samples) and the test set (data from the remaining 3 days, 63 samples). For the SVR, RFR, and BPNN models, the training set was expressed as a $1470 \times 10$ matrix, the test set was expressed as a $630 \times 10$ matrix. For the $1 D C N N$ and 1DCNN-RFR frameworks, the training set and the test set were obtained from all the 
response values and were expressed as a $147 \times 10 \times 100$ matrix and a $63 \times 10 \times 100$ matrix, respectively.

The SVR, RFR, and BPNN models, combined with SVs, were used to study the quantitative detection of adulteration in meat. The parameters searched for by the SVR model were the penalty factor $(1,5,10,20,50,100,200,500)$ and gamma $(0.01,0.1,1,5$, $10,20)$. The parameters searched for by the RFR model were the max depth $(3,5,7,9,11$, 13) and the minimum number of samples required to split an internal node (min. samples split: $7,14,21,28,35,42)$. Through the grid search skill, the penalty factor and gamma for the SVR model were set to 50 and 1, respectively. For the RFR model, the max depth and the min samples split were set to 5 and 35, respectively. For the BPNN model, the optimum network architecture was obtained with topological architecture 10-21-1. An MSE loss function serves to calculate error and is minimized by the stochastic gradient descent (SGD) optimizer. ReLU was selected as the activation function of the BPNN model. The regression results from the SVR, RFR, and BPNN models on the test set are shown in Table 5. Three evaluation metrics, the $R^{2}$, RMSE, and MAE, were computed to comprehensively and accurately assess these two regression models. When combined with SVs, the BPNN model achieved a marginally better result with an $R^{2}$ of 0.9456 , an RMSE of $4.6663 \%$, and a MAE of $2.6486 \%$. However, the performances of these three models were unsatisfactory because they failed to extract a sufficient number of features.

Table 5. Evaluation of the regressions from the five models using the test set in Experiment A using three metrics.

\begin{tabular}{cccc}
\hline Model & $\boldsymbol{R}^{\mathbf{2}}$ & RMSE (\%) & MAE (\%) \\
\hline SVR & 0.9183 & 5.7176 & 4.1394 \\
RFR & 0.9290 & 5.3293 & 2.9782 \\
BPNN & 0.9456 & 4.6663 & 2.6486 \\
1DCNN & 0.9852 & 2.4301 & 2.0250 \\
1DCNN-RFR & 0.9977 & 0.9491 & 0.4619 \\
\hline
\end{tabular}

The 10-channel input was submitted to the proposed 1DCNN framework consisting of the 1DCNN backbone and a 1DCNN regressor. The 1DCNN framework was implemented on a laptop (Intel Core i7-9750H processor). The number of epochs was 1100 and the training time was $57.6207 \mathrm{~s}$. The training loss of the 1DCNN framework during the training process is shown in Figure 6. The 1DCNN framework converged after 920 epochs. The parameters at 920 epochs were saved and used to evaluate the performance of the 1DCNN framework on the test set. As shown in Table 5, the 1DCNN framework performed much better than the SVR and RFR models, with an $R^{2}$ of 0.9852 , an RMSE of $2.4301 \%$, and a MAE of $2.0250 \%$ on the test set. The comparison with the SVR, RFR, and BPNN models, it was revealed that automatically mining a sufficient number of features from the E-nose data using the 1DCNN backbone significantly improved detection performance. 


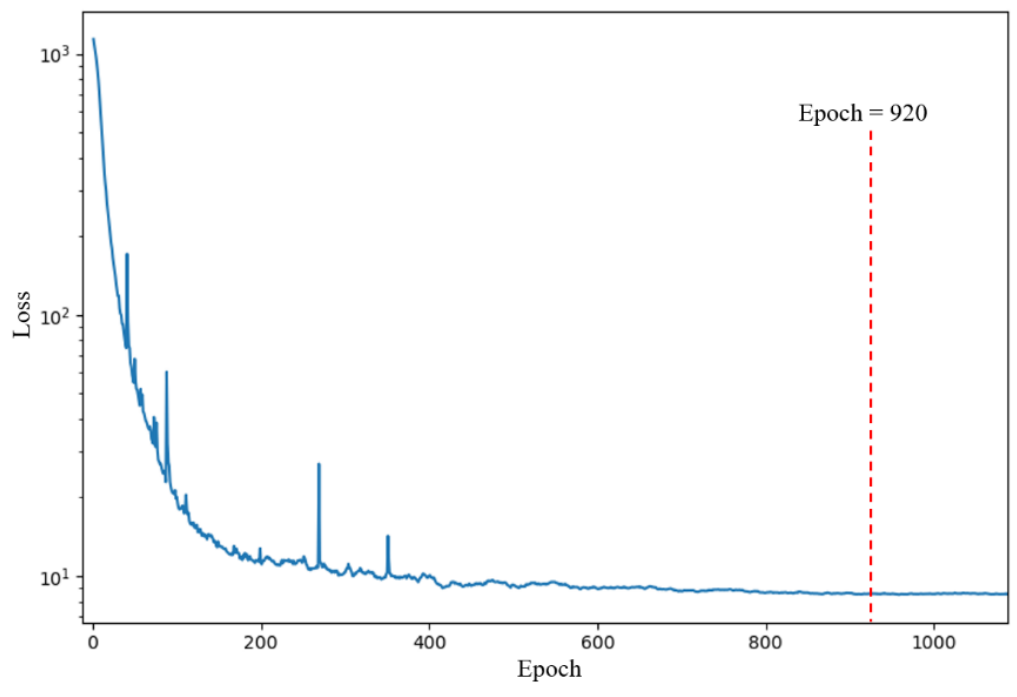

Figure 6. Training loss curve of the 1DCNN framework during training in Experiment A.

The 10-channel input was submitted to the proposed 1DCNN-RFR framework consisting of the 1DCNN backbone and the RFR. The 1DCNN backbone in the 1DCNN-RFR framework used the model parameters of the trained 1DCNN framework at 920 epochs. The training stage of the RFR in the 1DCNN-RFR framework was the same as that of the RFR model. Through the grid search skill, the max. depth and the min. samples split were set to 5 and 35, respectively. As shown in Table 5, the proposed 1DCNN-RFR framework achieved a better performance than all other models, with an $R^{2}$ of 0.9977 , an RMSE of $0.9491 \%$, and a MAE of $0.4619 \%$ on the test set. These results indicated that the strong prediction ability of the RFR improved the regression performance in the 1DCNN-RFR framework. The relationships between the predicted adulterated proportions by the five models and the corresponding actual adulterated proportions are shown in Figure 7. The $\mathrm{x}$-axis represents the sequence number of the tested samples, and the black and red points along the $y$-axis represent the actual and predicted proportions, respectively. Figure 7 intuitively illustrates that the predictive performances of models using the 1DCNN backbone to extract features were significantly better than those that did not use the 1DCNN backbone. The proposed 1DCNN-RFR framework achieved the best predictions and predicted almost all adulterated proportions precisely.

\subsubsection{Experiment $B$}

In practical applications, the number of samples will probably be much more limited than in Experiment A. Thus, Experiment B used a smaller number of training samples to further evaluate the generalization performance of the models. Dataset B was used in this experiment and was divided into the training set (data from the first 3 days, 63 samples) and the test set (data from the remaining 7 days, 147 samples). For the SVR, RFR, and BPNN models, the training set was expressed as a $630 \times 10$ matrix and the test set was expressed as a $1470 \times 10$ matrix. For the $1 D C N N$ and $1 D C N N-R F R$ frameworks, the training set and the test set were expressed as a $63 \times 10 \times 100$ matrix and a $147 \times 10 \times 100$ matrix, respectively. 


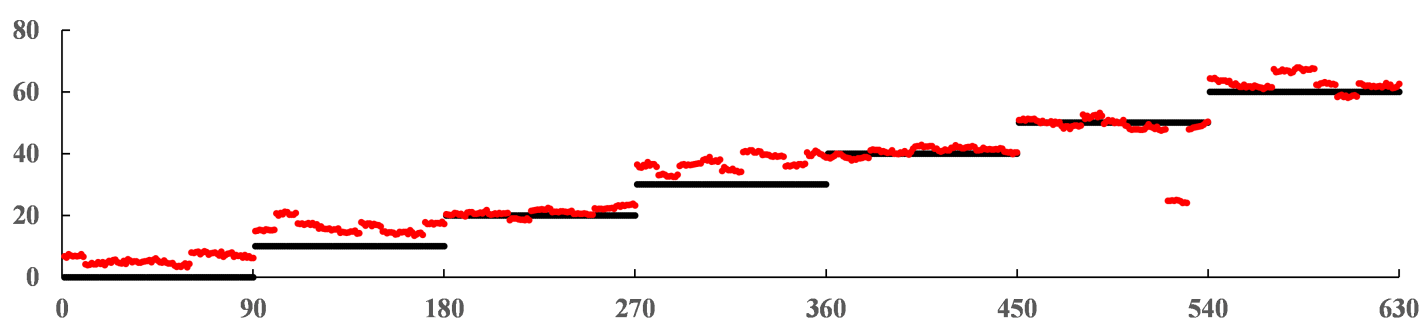

(a)

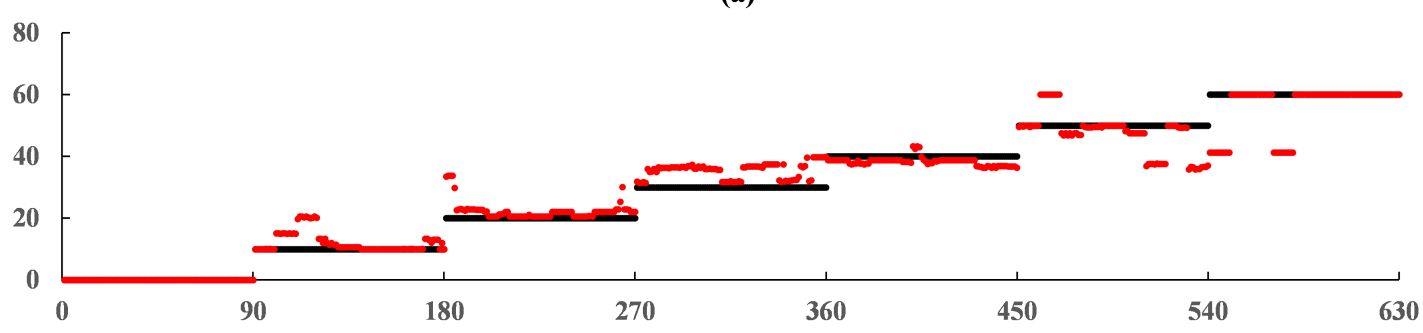

(b)

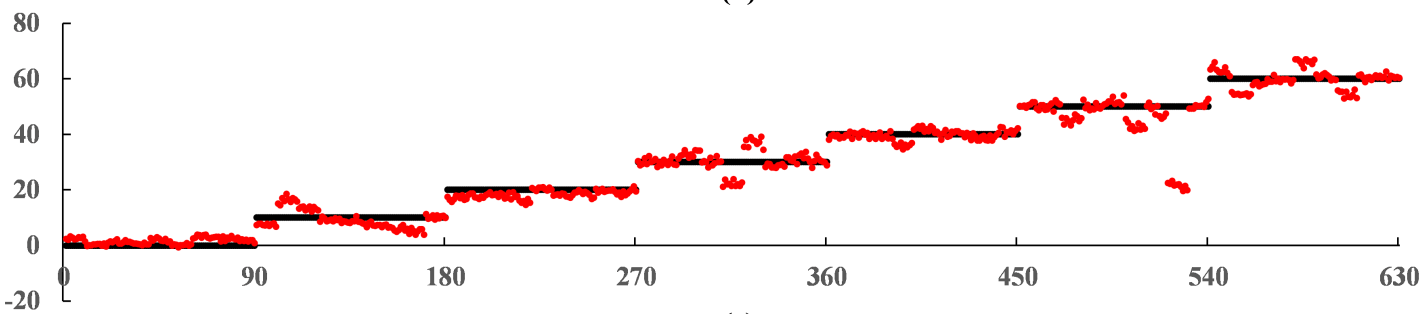

(c)
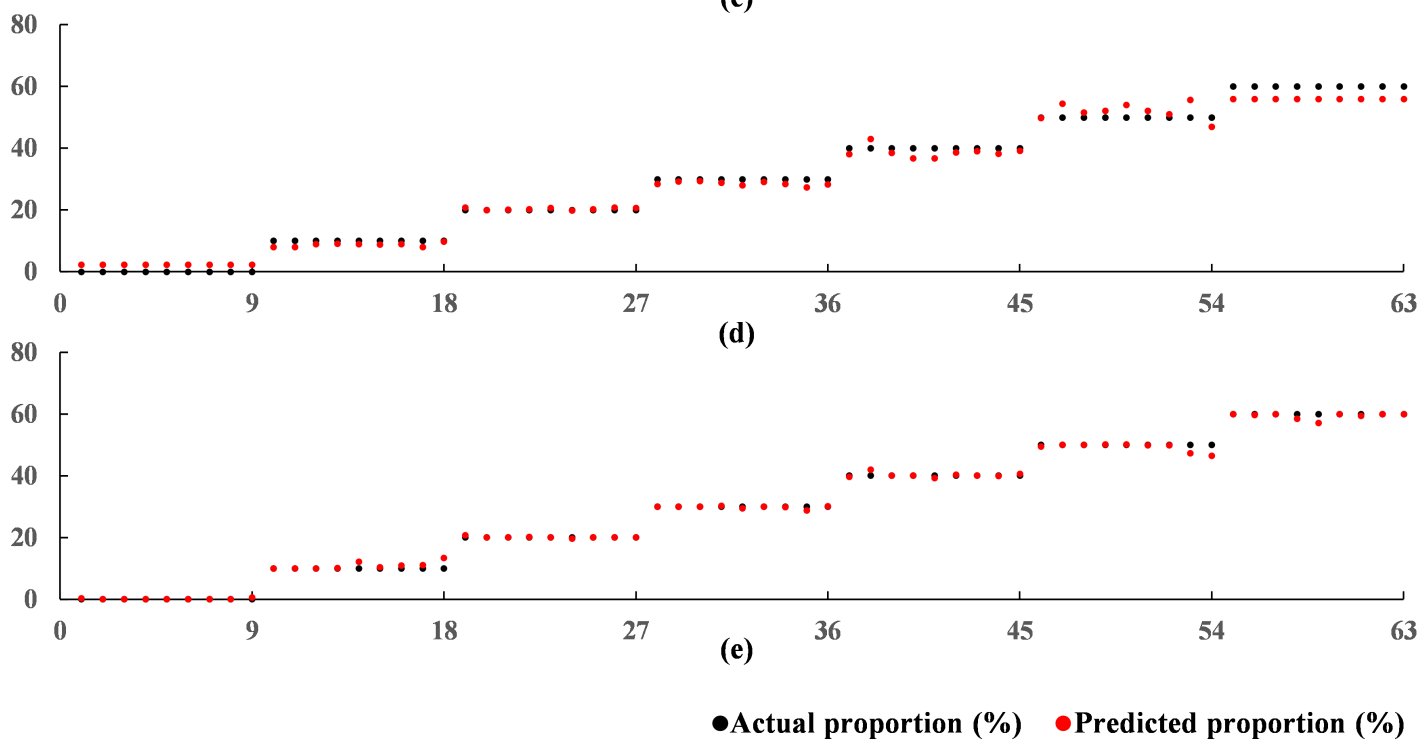

Figure 7. Relationships between the predicted adulterated proportions of the five models and the actual adulterated proportions in Experiment A: (a) SVR; (b) RFR; (c) BPNN; (d) 1DCNN; (e) 1DCNN-RFR.

All the experimental steps were the same as those of Experiment A, except for the 1DCNN where the batch size was adjusted from 49 to 21, while the other parameters were left unchanged. The best parameters of the SVR and RFR models were a penalty factor of 500, gamma of 0.1 , max. depth of 3, and min. samples split of 21. The max. depth and min. samples split of the RFR in the 1DCNN-RFR framework were set to 3 and 7, respectively. The training loss of the 1DCNN during training is shown in Figure 8. The 1DCNN framework converged after 980 epochs. The parameters at 980 epochs were saved and used to evaluate the performance of the 1DCNN framework on the test set. The test set regression results from the five models (the SVR model, RFR model, BPNN 
model, 1DCNN framework, and 1DCNN-RFR framework) are shown in Table 6. The prediction performances of the SVR and RFR models in Experiment B were much worse than those of Experiment A. The BPNN model, the 1DCNN framework, and the proposed 1DCNN-RFR framework also suffered a slight reduction in performance. Even so, the 1DCNN framework and the 1DCNN-RFR framework performed much better than the SVR and RFR models. The 1DCNN-RFR model still worked best and obtained a good result with an $R^{2}$ of 0.9858 , an RMSE of $2.3849 \%$, and a MAE of $1.1625 \%$ on the test set. The regression results in Experiment $B$ further demonstrated the superiority of the proposed 1DCNN-RFR framework, which performed well despite the much smaller sample size. The relationships between the predicted adulterated proportions of the five models and the actual adulterated proportions are shown in Figure 9. These relationships showed that the SVR and RFR models, which did not use the 1DCNN backbone and were unable to extract a sufficient number of features, had extremely poor prediction results. The proposed 1DCNN-RFR framework performed best.

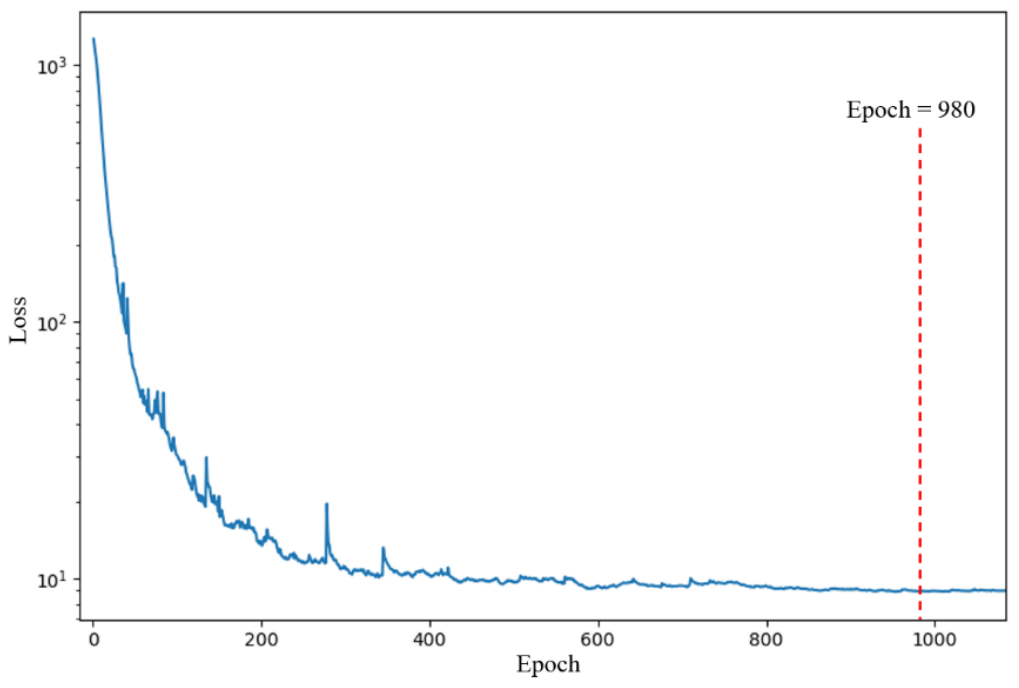

Figure 8. Training loss curve of the 1DCNN framework during training in Experiment B.

Table 6. Evaluation of regressions on the test set by the five models in Experiment B using three metrics.

\begin{tabular}{cccc}
\hline Model & $\boldsymbol{R}^{\mathbf{2}}$ & RMSE (\%) & MAE (\%) \\
\hline SVR & 0.6108 & 12.4770 & 9.7819 \\
RFR & 0.7734 & 9.5204 & 5.6891 \\
BPNN & 0.9219 & 5.5910 & 4.2554 \\
1DCNN & 0.9703 & 3.4441 & 2.6853 \\
1DCNN-RFR & 0.9858 & 2.3849 & 1.1625 \\
\hline
\end{tabular}




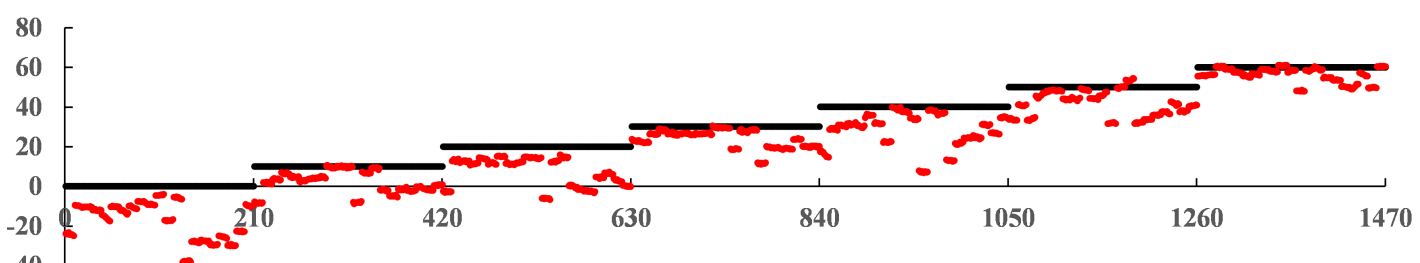

(a)
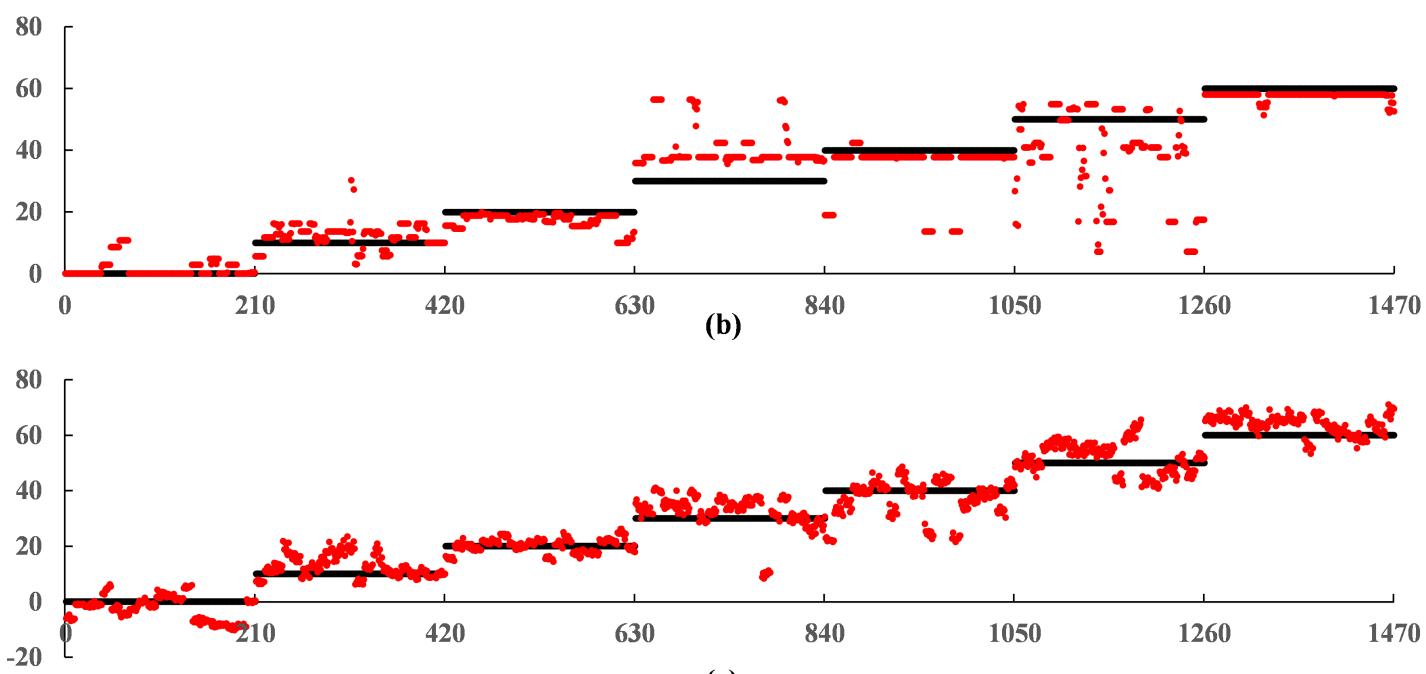

(c)
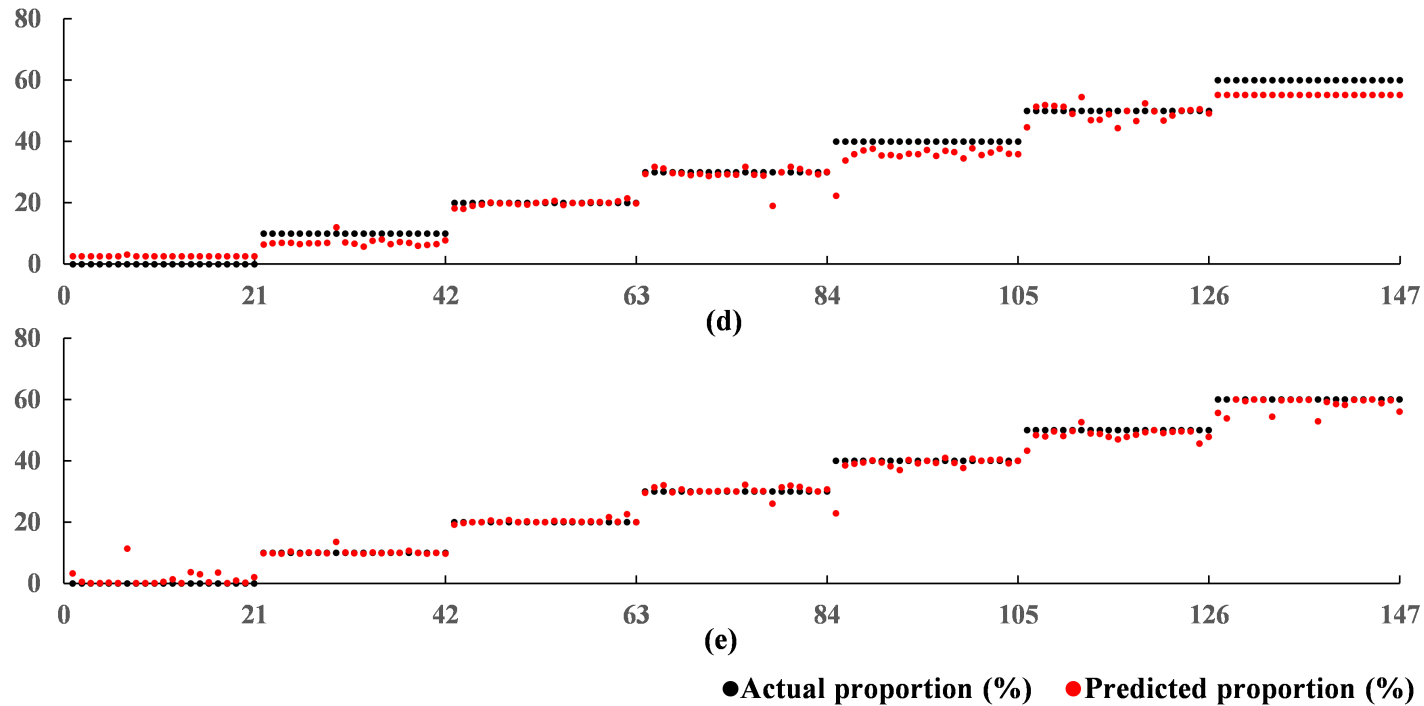

Figure 9. Relationships between the predicted adulterated proportions of the five models and the actual adulterated proportions in Experiment B. (a) SVR; (b) RFR; (c) BPNN; (d) 1DCNN; (e) 1DCNN-RFR.

\section{Conclusions}

In this study, a novel framework 1DCNN-RFR, consisting of a 1DCNN backbone and an RFR, was proposed for the quantitative detection of beef adulterated with pork using an MOS-based E-nose. The 1DCNN backbone automatically extracted a sufficient number of features from the E-nose data. The RFR strengthened the generalization ability of the 1DCNN framework and improved the prediction performance. Compared with the other four models (SVR, RFR, BPNN, and 1DCNN), the proposed 1DCNN-RFR framework obtained the best results on the test set, with an $R^{2}$ of 0.9977 , an RMSE of $0.9491 \%$, and a MSE of $0.4619 \%$. All the findings suggest that the MOS-based E-nose coupled with the 
proposed 1DCNN-RFR framework has great potential for the quantitative detection of minced beef adulterated with pork.

Author Contributions: Conceptualization, C.H. and Y.G.; methodology, C.H. and Y.G.; validation, C.H. and Y.G.; formal analysis, C.H.; writing-original draft preparation, C.H.; writing-review and editing, Y.G.; visualization, Y.G.; supervision, Y.G.; funding acquisition, Y.G. All authors have read and agreed to the published version of the manuscript.

Funding: This research was funded by the National Natural Science Foundation of China [Grant No. 61876059].

Institutional Review Board Statement: Not applicable.

Informed Consent Statement: Not applicable.

Data Availability Statement: The data presented in this study are available at Figshare (https: //doi.org/10.6084/m9.figshare.19200284.v1).

Conflicts of Interest: The authors declare no conflict of interest.

\section{Abbreviations}

The following abbreviations are used in this manuscript:

$\begin{array}{ll}\text { E-nose } & \text { Electronic Nose } \\ \text { MOS } & \text { Metal Oxide Semiconductor } \\ \text { RF } & \text { Random Forest } \\ \text { RFR } & \text { Random Forest Regressor } \\ \text { SVR } & \text { Support Vector Regression } \\ \text { CNN } & \text { Convolutional Neural Network } \\ \text { 1DCNN } & \text { One-dimensional CNN } \\ \text { 1DCNN-RFR } & \text { a 1DCNN backbone combined with an RFR } \\ \text { PCA } & \text { Principal Component Analysis } \\ \text { RBF } & \text { Radial Basis Function } \\ R^{2} & \text { the Coefficient of Determination } \\ \text { RMSE } & \text { the Root Mean Square Error } \\ \text { MAE } & \text { the Mean Absolute Error } \\ \text { SV } & \text { Stable Value } \\ \text { SGD } & \text { Stochastic Gradient Descent } \\ \text { PC1 } & \text { Principal Component 1 } \\ \text { PC2 } & \text { Principal Component 2 } \\ \text { PC3 } & \text { Principal Component 3 }\end{array}$

\section{References}

1. Rahmati, S.; Julkapli, N.M.; Yehye, W.A.; Basirun, W.J. Identification of meat origin in food products-A review. Food Control 2016, 68, 379-390. [CrossRef]

2. OECD/FAO. OECD-FAO Agricultural Outlook 2021-2030; Technical Report OECD/FAO; OECD/FAO: Paris, France, 2021 [CrossRef]

3. Rohman, A. The employment of Fourier transform infrared spectroscopy coupled with chemometrics techniques for traceability and authentication of meat and meat products. J. Adv. Vet. Anim. Res. 2019, 6, 9-17. [CrossRef] [PubMed]

4. Chen, X.; Lu, L.; Xiong, X.; Xiong, X.; Liu, Y. Development of a real-time PCR assay for the identification and quantification of bovine ingredient in processed meat products. Sci. Rep. 2020, 10, 2052. [CrossRef]

5. Li, X.; Guan Y. Specific Identification of the Adulterated Components in Beef or Mutton Meats Using Multiplex PCR. J. AOAC Int. 2019, 102, 1181-1185. [CrossRef] [CrossRef]

6. Li, J.; Wei, Y.; Li, J.; Liu, R.; Xu, S.; Xiong, S.; Guo, Y.; Qiao, X.; Wang, S. A novel duplex SYBR Green real-time PCR with melting curve analysis method for beef adulteration detection. J. Abbr. 2021, 338, 127932. [CrossRef] [PubMed]

7. Uddin, S.M.K.; Hossain, M.A.M.; Chowdhury, Z.Z.; Johan, M.R. Detection and discrimination of seven highly consumed meat species simultaneously in food products using heptaplex PCR-RFLP assay. J. Food Compos. Anal. 2021, 100, 103938. [CrossRef]

8. Jawla, J.; Kumar, R.R.; Mendiratta, S.K.; Agarwal, R.K.; Singh, P.; Saxena, V.; Kumari, S.; Boby, N.; Kumar, D.; Rana, P. Onsite paper-based Loop-Mediated Isothermal Amplification coupled Lateral Flow Assay for pig tissue identification targeting mitochondrial CO I gene. J. Food Compos. Anal. 2021, 102, 104036. [CrossRef] 
9. Thienes, C.P.; Masiri, J.; Benoit, L.A.; Barrios-Lopez, B.; Samuel, S.A.; Cox, D.P.; Dobritsa, A.P.; Nadala, C.; Samadpour, M. Quantitative Detection of Horse Contamination in Cooked Meat Products by ELISA. J. AOAC Int. 2018, 101, 817-823. [CrossRef]

10. Edwards, K.; Manley, M.; Hoffman, L.C.; Williams, P.J. Non-Destructive Spectroscopic and Imaging Techniques for the Detection of Processed Meat Fraud. Foods 2021, 10, 448. [CrossRef]

11. Jiang, H.; Wang, W.; Zhuang, H.; Yoon, S.C.; Yang, Y.; Zhao, X. Hyperspectral imaging for a rapid detection and visualization of duck meat adulteration in beef. Food Anal. Methods 2019, 12, 2205-2215. [CrossRef]

12. Nurjuliana, M.; Man, Y.B.C.; Hashim, D.M.; Mohamed, A.K.S. Rapid identification of pork for halal authentication using the electronic nose and gas chromatography mass spectrometer with headspace analyzer. Meat Sci. 2011, 88, 638-644. [CrossRef] [PubMed]

13. Zhang, Y.; Liu, M.; Wang, S.; Kang, C.; Zhang, M.; Li, Y. Identification and quantification of fox meat in meat products by liquid chromatography-tandem mass spectrometry. Food Chem. 2022, 372, 131336. [CrossRef] [PubMed]

14. Tian, X.; Wang, J.; Cui, S. Analysis of pork adulteration in minced mutton using electronic nose of metal oxide sensors. J. Food Eng. 2013, 119, 744-749. [CrossRef]

15. Leng, T.; Li, F.; Xiong, L.; Xiong, Q.; Zhu, M.; Chen, Y. Quantitative detection of binary and ternary adulteration of minced beef meat with pork and duck meat by NIR combined with chemometrics. Food Control 2020, 113, 107203. [CrossRef]

16. Chen, Z.; Wu, T.; Xiang, C.; Xu, X.; Tian, X. Rapid Identification of Rainbow Trout Adulteration in Atlantic Salmon by Raman Spectroscopy Combined with Machine Learning. Molecules 2019, 24, 2851. [CrossRef] [PubMed]

17. Jiang, H.; Cheng, F.; Shi, M. Rapid Identification and Visualization of Jowl Meat Adulteration in Pork Using Hyperspectral Imaging. Foods 2020, 9, 154. [CrossRef]

18. Boeker, P. On 'Electronic Nose' methodology. Sens. Actuators B 2014, 204, 2-17. [CrossRef]

19. Liu, H.; Yu, D.; Gu, Y. Classification and evaluation of quality grades of organic green teas using an electronic nose based on machine learning algorithms. IEEE Access 2019, 7, 172965-172973. [CrossRef]

20. Han, F.; Huang, X.; Aheto, J.H.; Zhang, D.; Feng, F. Detection of Beef Adulterated with Pork Using a Low-Cost Electronic Nose Based on Colorimetric Sensors. Foods 2020, 9, 193. [CrossRef]

21. Sarno, R.; Triyana, K.; Sabilla, S.I.; Wijaya, D.R.; Sunaryono, D.; Fatichah, C. Detecting Pork Adulteration in Beef for Halal Authentication Using an Optimized Electronic Nose System. IEEE Access 2020, 8, 221700-221711. 043394. [CrossRef]

22. GB 20799-2016; National Food Safety Standard-Operating Hygienic Code for Meat and Meat Products. Available online: http:/ / www.shanglin.gov.cn/gk/xxgkml/zdlyxxgk/aqsc/t1358084.html (accessed on 24 November 2021).

23. Yu, D.; Gu, Y. A Machine Learning Method for the Fine-Grained Classification of Green Tea with Geographical Indication Using a MOS-Based Electronic Nose. Foods 2021, 10, 795. [CrossRef] [PubMed]

24. Wei, H.; Gu, Y. A Machine Learning Method for the Detection of Brown Core in the Chinese Pear Variety Huangguan Using a MOS-Based E-Nose. Sensors 2020, 20, 4499. [CrossRef]

25. Cao, L.J.; Chua, K.S.; Chong, W.K.; Lee, H.P.; Gu, Q.M. A comparison of PCA, KPCA and ICA for dimensionality reduction in support vector machine. Neurocomputing 2003, 55, 321-336. [CrossRef]

26. Liu, H.; Li, Q.; Yan, B.; Zhang, L.; Gu, Y. Bionic Electronic Nose Based on MOS Sensors Array and Machine Learning Algorithms Used for Wine Properties Detection. Sensors 2019, 19, 45. [CrossRef] [PubMed]

27. Yu, H.; Chen, R.; Zhang, G. A SVM Stock Selection Model within PCA. Procedia Comput. Sci. 2014, 31, 406-412. [CrossRef]

28. Yu, D.; Wang, X.; Liu, H.; Gu, Y. A Multitask Learning Framework for Multi-Property Detection of Wine. IEEE Access 2019, 7 , 123151-123157. [CrossRef]

29. LeCun, Y.; Bengio, Y.; Hinton, G. Deep learning. Nature 2015, 521, 436-444. [CrossRef]

30. Gu, J.; Wang, Z.; Kuen, J.; Ma, L.; Shahroudy, A.; Shuai, B.; Liu, T.; Wang, X.; Wang, G.; Cai, J.; et al. Recent advances in convolutional neural networks. Pattern Recog. 2018, 77, 354-377. [CrossRef]

31. Wei, H.; Zhang, Q.; Shang, M.; Gu, Y. Extreme learning Machine-based classifier for fault diagnosis of rotating Machinery using a residual network and continuous wavelet transform. Measurement 2021, 183, 109864. 109864. [CrossRef]

32. Liang, S.; Liu, H.; Gu, Y.; Guo, X.; Li, H.; Li, L.; Wu, Z.; Liu, M.; Tao, L. Fast automated detection of COVID-19 from medical images using convolutional neural networks. Commun. Biol. 2021, 4, 35. [CrossRef]

33. Breiman, L. Random Forests. Mach. Learn. 2001, 45, 5-32. [CrossRef]

34. Liu, Z.; Gilbert, G.; Cepeda, J.M.; Lysdahl, A.O.K.; Piciullo, L.; Hefre, H.; Lacasse, S. Modelling of shallow landslides with machine learning algorithms. Geosci. Front. 2021, 12, 385-393. [CrossRef]

35. Kensert, A.; Alvarsson, J.; Norinder, U.; Spjuth, O. Evaluating parameters for ligand-based modeling with random forest on sparse data sets. J. Cheminform. 2018, 10, 49. [CrossRef]

36. Liaw, A.; Wiener, M. Classification and regression by random Forest. R News 2002, 1, 18-22.

37. Wang, X.; Gu, Y.; Liu, H. A Transfer Learning Method for the Protection of Geographical Indication in China Using an Electronic Nose for the Identification of Xihu Longjing Tea. IEEE Sens. J. 2021, 21, 8065-8077. [CrossRef]

38. Sun, Y.; Zhang, H.; Zhao, T.; Zou, Z.; Shen, B.; Yang, L. A New Convolutional Neural Network with Random Forest Method for Hydrogen Sensor Fault Diagnosis. IEEE Access 2020, 8, 85421-85430. [CrossRef] 
39. Renaud, O.; Victoria-Feser, M.-P. A robust coefficient of determination for regression. J. Stat. Plan. Inference 2010, 140, 1852-1862. [CrossRef]

40. Wang, Y.; Huang, S.; Dai, J.; Tang, J. A Novel Bearing Fault Diagnosis Methodology Based on SVD and One-Dimensional Convolutional Neural Network. Shock Vib. 2020, 2020, 1850286. [CrossRef]

41. Huang, S.; Tang, J.; Dai, J.; Wang, Y. Signal Status Recognition Based on 1DCNN and Its Feature Extraction Mechanism Analysis. Sensors 2019, 19, 2018. [CrossRef]

42. Ye, Y.; Gao, J.; Shao, Y.; Li, C.; Jin, Y.; Hua, X. Robust support vector regression with generic quadratic nonconvex $\varepsilon$-insensitive loss. Appl. Math. Model. 2020, 82, 235-251. [CrossRef] 